\title{
A method for determining in 3-D, the true dip and azimuth of a reflecting horizon
}

\author{
Brian J. Evans*, Norman F. Uren, Curtin University, Australia; and John A. McDonald, \\ Curtin University and University of Houston
}

\section{SUMMARY}

A 3-D field method has been devised in which the true dip of a reflecting horizon and its azimuth can be measured within a survey area. The method requires a suitable horizon to which the velocity is known, and assumes that the reflecting horizon has constant dip throughout the area. Two orthogonal swath surveys are performed, and data are stacked using variable dip and strike values. A 3-D NM0 equation corrects data to a zero-offset reference point. An optimum value of dip and strike provides the best reflection line-up at the reference point, producing the value of true dip and its azimuth for that horizon. A physical model was used to demonstrate the method, which was able to predict dips and their azimuths to within half a degree.

\section{Introduction}

3-D seismic stacking procedures normally assume a single stacking velocity for all traces in a bin. However, a change in azimuth from source to receiver changes the apparent dip and the stacking velocity. Levin (1971) discussed how 3-D dip can affect 2-D inline data, and concluded that for dips of less than 10 degrees, the NM0 stacking velocity was approximately the same as the velocity of the medium. Since most sedimentary strata have dips of less than 10 degrees, that assumption has been adequate.

A 3-D NM0 expression separates the velocity term from the dip term for any value of dip, for a single reflection plane. Using this expression, the apparent dip and azimuth can be determined for each source/receiver pair, so that the 3-D NM0 equation can then be used to stack swath mid-point data without the need for conventional velocity analysis.

\section{Dip Prediction Concept}

The apparent dip between each source and receiver pair in any CMP bin changes the stacking velocity for each trace in that bin. If it were possible to align the arrivals for a given reflecting horizon across all binned traces with that for a zero-offset reference point, then it should be possible to determine the true dip and azimuth of the reflecting horizon. Then the 3-D NM0 expression may be used to correctly stack bin data if the variable apparent dip is accounted for.

\section{Data Acquisition}

To test the concept of dip detection, a physical model was constructed from Plexiglas. A 5.50 dipping plane was machined into the block's base, as shown in Figure 1, using a length scale of I:20 000. A reference point was chosen at location '0' on the model. A 2300 $\mathrm{m}$ source line was positioned parallel to and $2300 \mathrm{~m}$ apart from a receiver line (scaled distances). Using $450 \mathrm{MHz}$ ultrasonic transducers, a CMP data set was collected along a line parallel to the dip direction. 24 shots at $100 \mathrm{~m}$ intervals were fired into 24 receivers, the receivers being positioned in a static spread while the shots were rolled-through. Because most ray paths were across the dip direction, this form of recording was termed 'cross-dip' recording. The dip direction angle was given the symbol $\mathrm{a}$, and the strike angle was given the symbol $\beta$ and in this case of course, $\beta=0$ degrees.

The model was rotated through $90^{\circ}$ and the acquisition repeated but in this direction, most of the ray paths were in the 'down-dip' direction.

\section{Processing and Interpretation}

A suite of shot records for the 'cross-dip' survey is shown in Figure 2. The first arrival is the reflection from the model's base, and arrivals after this are surface waves and multiples. The cross-dip records show the effect of using a static receiver spread while the source rolls along the shot line. The reflection moves down in time from around $620 \mathrm{~ms}$ on the left to $680 \mathrm{~ms}$ on the right, indicating that the reflection is dipping from left to right.

A set of CMP bin gathers are shown in Figure 3 for the cross-dip survey. As expected, the gathers have a lower number of traces at each end of the display than in the center of the display (CMP 24), where it has 24 traces. These data were now ready for 3-D NM0 application.

The geometry for each source/receiver pair was known, as well as the velocity of the medium (2 666 $\mathrm{m} / \mathrm{s}$ ). The data were displayed with mid-point gather 24 having the most traces alongside the stack of the binned traces of the mid-point line. An example of this for the cross-dip recording configuration is shown in Figure 4. Using an a value of $5.5^{\circ}$, and a $\beta$ value of zero, the reflection events align within the gather of CMP 24 and along the mid-point line. 
To the right of the display in Figure 4, is the stacked response of all the traces along the line. Such a stacked response may be used as numerical support for the optimum line-up of reflections. The peak-topeak stacked response value for an a of $5.5^{\circ}$, and $\beta$ of zero, was 82. A value for a of $4^{\circ}$ and $\beta$ of zero was then applied, with the result shown in Figure 5 . The peak-to-peak value was 56 . Using an a of $4^{\circ}$ and $\beta$ of zero made the reflection dip upwards in CMP bin 24, and dip downwards along the line. In Figure 6, an a of $6^{\circ}$ and $\beta$ of zero caused the CMP binned data to appear horizontal but the stacked line dips slightly upwards. The peak-to-peak response therefore was 77.8 , indicating dip and strike values close to optimum. As can be seen then the method optimises the value of the dip to within a half degree.

The down-dip results using the orthogonal data set were identical. Eventually, the model was rotated $45^{\circ}$ and values of a and $\beta$ were determined for each bin as $3.9^{\circ}$ This computed to a true dip of 5.50 at an azimuth of $45^{\circ}$ with respect to the recording configuration.

\section{Discussion}

This method of true dip and azimuth detection has used a physical model to test its feasibility. The model was isotropic, had a known velocity and subsequent tests applying different velocities in the 3-D NMO equation did not affect the accuracy of the computational process. Using the opposite corner of the model as the reference point worked equally well, showing that the reference point may be arbitrarily selected. A change in the depth of the reflection at the reference point made no difference, since changing depth only bulk shifted the data set.

A major factor in this research has been the application of the 3-D NMO equation which is dependent upon the true dip and its azimuth (represented in the equation by apparent dip in two orthogonal directions), and the velocity of the medium

Advantages of this method are:

(i) Prior knowledge of the magnitude and direction of true dip determined by the method enables the subsequent survey lines to be placed in the dip direction.

(ii) The stacking velocity is no longer dip dependent and a constant velocity could be used for stacking bin data, with dip as a separate factor.

(iii) With the dip for any azimuth known, the velocity of the medium is constant, and low fold gathers may be easily stacked without the attendant problems of determining stacking velocity.

Future work will involve the 3-D NMO solution for a second layer, continuing downwards in a layer stripping manner, so that the method of dip detection can be applied to a multi-layered environment.

\section{Conclusions}

A method has been shown to determine the true dip and its azimuth of a single reflecting horizon. Input demanded by the method includes a knowledge of the velocity of the medium and the depth normal to the reflection, at a reference location. Also, the geometry of the source and receiver lines with respect to the reference point must be known.

\section{References}

Levin, F.K., 1971, Apparent velocity from dipping interface reflections: Geophysics, 36, 51 0-51 5.

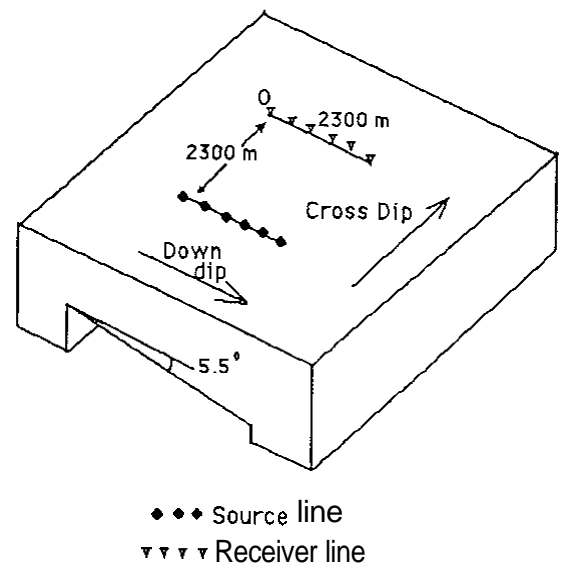

Figure 1. Physical model used to test the dip detection process. Cross-dip recording configuration is shown. Distances were scaled 1: 20000. 
True dip and azimuth of a reflecting horizon

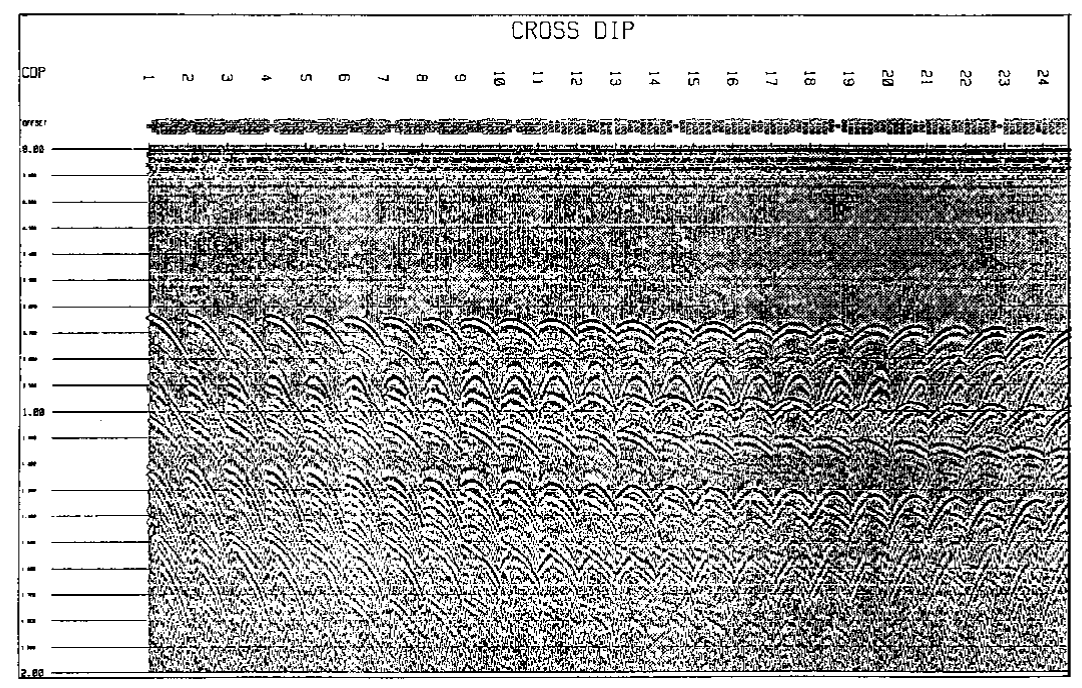

Figure 2. Cross-dip shot records over the model.

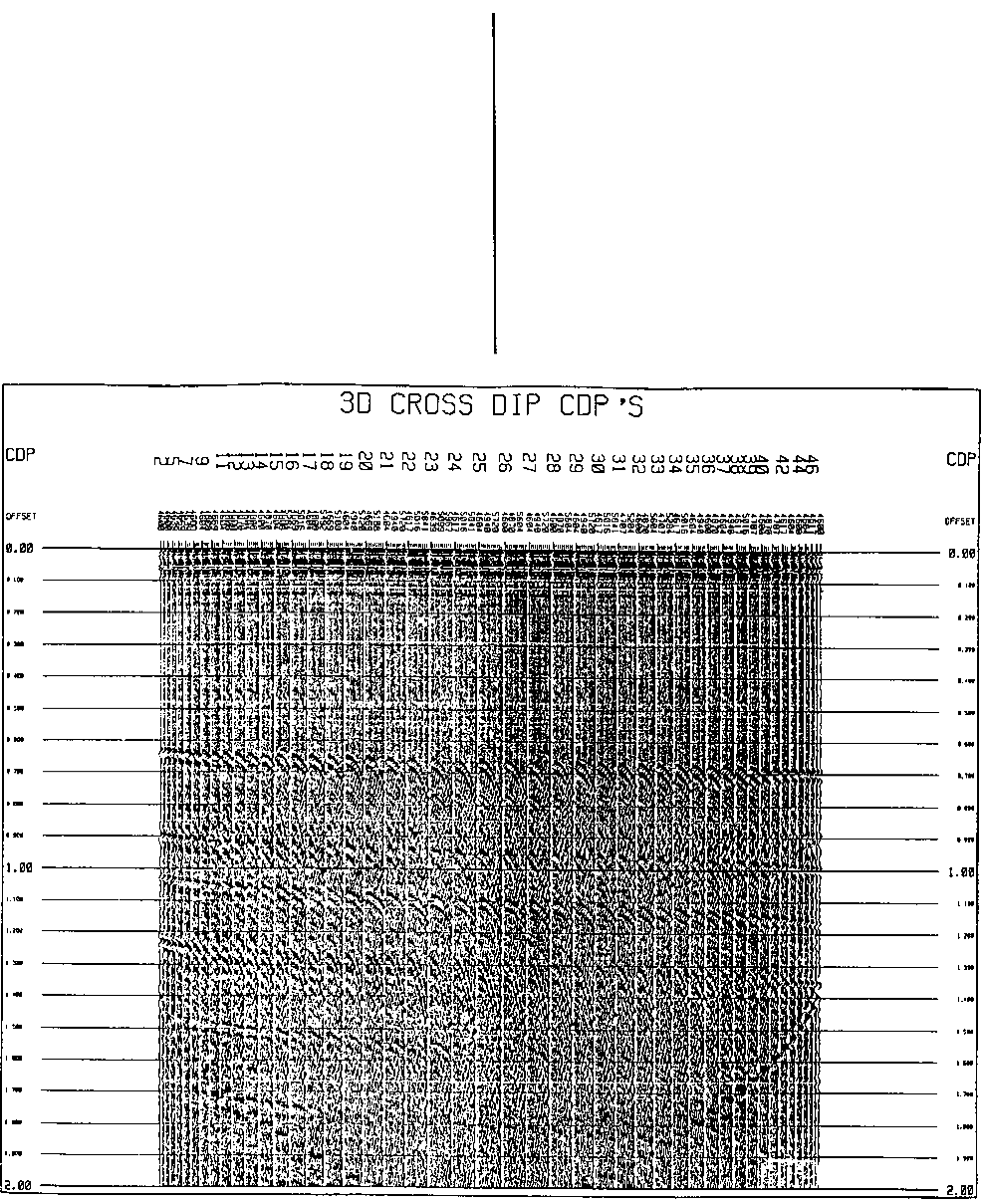

Figure 3. Cross-dip CDP binned data. 

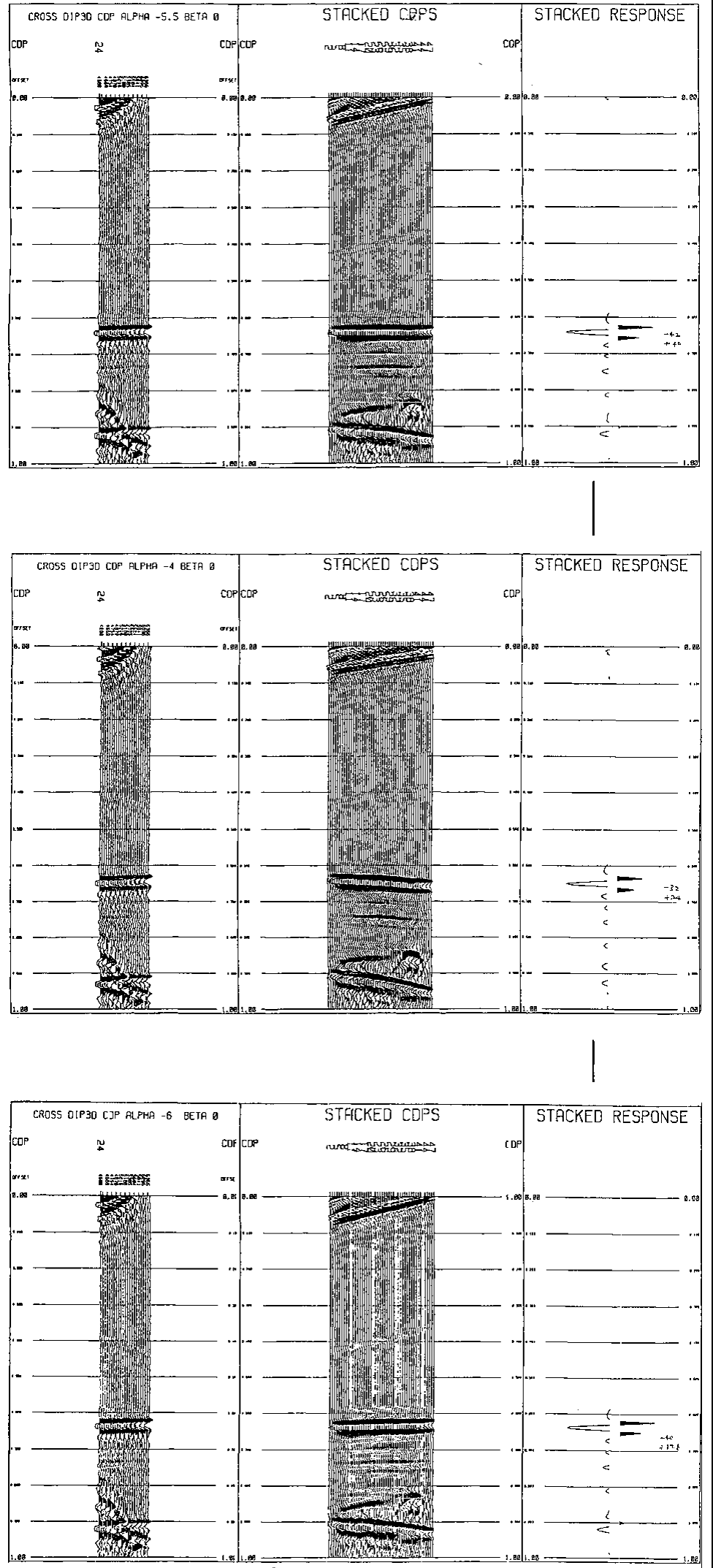

Figure 4. Cross-dip section with using a $=55^{\circ}$ and $\beta=0^{\circ}$, produces a horizontal binned gather and horizontal stacked line, with a peak-to-peak stacked response of 82 . This maximum value indicates optimum dip values.

Figure 5. Cross-dip section with using a $=4.0^{\circ}$ and $\beta=0^{\circ}$, produces a dipping binned gather and a dipping stacked line, with a peak-to-peak stacked response of 56 .

Figure 6. Cross-dip section with using a $=6.0^{\circ}$ and $\beta=0^{\circ}$, produces a horizontal binned gather but a dipping stacked line with a peak-to-peak stacked response of 77.8. This high response value indicates almost correct dip and strike values. 